\title{
A THEOREM ON SCHLICHT FUNCTIONS
}

\section{S. B. TOWNES}

In 1946 Friedman [2] found all schlicht functions, with certain restrictions, that have rational integral coefficients. It is the purpose of this article to find all schlicht functions similarly restricted, whose $n$th powers, $n$ a positive integer, have coefficients belonging to an integral domain $I$, of characteristic zero, and that has no integer except zero of absolute value less than 1 .

1. Notations and definitions. A function is said to be schlicht in a domain $D$ if for any two points $z_{1}$ and $z_{2}$, belonging to $D$, we have $f\left(z_{1}\right)=f\left(z_{2}\right)$ only if $z_{1}=z_{2}$. We shall seek all functions

$$
f(z)=\sum_{1}^{\infty} a_{i} z^{i}=z+a_{2} z^{2}+a_{3} z^{3}+\cdots
$$

which are regular and schlicht within the circle $|z|<1$ and such that $g(z)=f^{n}(z)=\sum_{1}^{\infty} b_{i} z^{n+i}=z^{n}+b_{1} z^{n+1}+b_{2} z^{n+2}+\cdots$ have coefficients belonging to $I$. Let $h(z)=z^{n} / g(z)$ have the expansion

$$
\sum_{0}^{\infty} c_{i} z^{i}=1+c_{1} z+c_{2} z^{2}+\cdots .
$$

\section{Preliminary lemmas.}

Lemma 1. If $g(z)$ has coefficients belonging to $I$, then $h(z)$ is a polynomial with coefficients belonging to $I$, and conversely.

The relationship between the coefficients of $h(z)$ and those of $g(z)$ is

$$
\begin{aligned}
& b_{1}+c_{1}=0, \\
& b_{2}+b_{1} c_{1}+c_{2}=0, \\
& \cdot \cdot \cdot \cdot \cdot \cdot \cdot \cdot \cdot \cdot, \\
& b_{m}+b_{m-1} c_{1}+b_{m-2} c_{2}+\cdots+c_{m}=0 .
\end{aligned}
$$

If the $b_{i}$ are integers of $I$, the $c_{i}$ are also, and conversely.

Let

$$
[f(z) / z]^{-\alpha / 2}=1+\sum_{1}^{\infty} k_{i} z^{i}
$$

The Prawitz inequality [3], as revised by Bernardi [1], is

Received by the editors February 13, 1953 and, in revised form, April 29, 1953. July 30, 1953, and January 13, 1954. 


$$
\sum_{1}^{\infty} \frac{2 m-\alpha}{\alpha}\left|k_{m}^{2}\right| \leqq 1
$$

For $\alpha=2 n$ this becomes

$$
\begin{aligned}
\sum_{1}^{\infty} i\left|c_{n+i}^{2}\right| & =\left|c_{n+1}^{2}\right|+2\left|c_{n+2}^{2}\right|+\cdots \\
& \leqq n+(n-1)\left|c_{1}^{2}\right|+(n-2)\left|c_{2}^{2}\right|+\cdots+\left|c_{n-1}^{2}\right|
\end{aligned}
$$

The right side of (2.2) has a finite number of terms, and is therefore finite. By (2.1) the $c_{i}$ are integers of $I$. It follows from (2.2) and the restrictions on $I$ that the greatest value of $i$ for which $c_{i}$ may be different from zero is finite.

Lemma 2. All the zeros of the polynomial $h(z)$ lie on the circle $|z|=1$. The constant term of $h(z)$ is 1 and all its coefficients are integers of $I$, and therefore either zero or not less than 1 in absolute value. It follows that if any zero of $h(z)$ is different from 1 in absolute value, there is at least one which is less than 1 in absolute value. This is contrary to the hypothesis that $f(z)$, which is equal to $z /[h(z)]^{1 / n}$, is regular for $|z|<1$.

Lemмa 3. The degree of $h(z)$ does not exceed $2 n$.

Let

$$
h(z)=c_{m} z^{m}+c_{m-1} z^{m-1}+\cdots+1, \quad \text { where }\left|c_{m}\right|=1 .
$$

Then

$$
f^{\prime}(z)=\frac{(m-n) c_{m} z^{m}+(m-n-1) c_{m-1} z^{m-1}+\cdots-n}{n[h(z)]^{(n+1) / n}} .
$$

If $m>2 n$, then $n /(m-n)$, the absolute value of the product of the finite zeros of $f^{\prime}(z)$, is less than 1. It follows that $f^{\prime}(z)$ has at least one zero for $|z|<1$ and by Bernardi [1], $f(z)$ is not schlicht.

\section{The main result.}

THEOREM. The set of functions $f(z)$, defined in $\$ 1$, consists of $z$ and all the functions $z /\left[(h(z)]^{1 / n}\right.$ in which $h(z)$ is a polynomial with coeffcients in I satisfying the restrictions of Lemmas 2 and 3.

It remains to prove the functions $z /[h(z)]^{1 / n}$ are schlicht. First, consider the variation of the function

$$
f_{1}(z)=\frac{z}{(z-1)^{2}}
$$


as $z$ goes around the unit circle indented at $z=1$. Put $z=e^{i \theta}$. For $\epsilon<\theta<2 \pi-\epsilon$ then

$$
f_{1}\left(e^{i \theta}\right)=\frac{-1}{4 \sin ^{2} \theta / 2} .
$$

As $\theta$ goes from $\epsilon$ to $2 \pi-\epsilon$ the argument of $f_{1}(z)$ remains equal to $\pi$. On the indentation we have $z=1-e^{i \phi}$ where $\pi / 2<\phi<3 \pi / 2$. But then

$$
f_{1}(z)=\frac{1-\epsilon e^{i \phi}}{\epsilon^{2} e^{2 i \phi}}
$$

and for sufficiently small $\epsilon$ the argument of $f_{1}(z)$ is $-2 \phi$. As $\phi$ goes from $3 \pi / 2$ to $\pi / 2$, the argument of $f_{1}(z)$ increases by $2 \pi$. In short, the argument of $f_{1}(z)$ along the indented circle is nondecreasing with a total variation of $2 \pi$. The same is true for the argument of the function

$$
\frac{z}{\left(z-e^{i \alpha}\right)^{2}}=e^{-i \alpha} f_{1}\left(z e^{-i \alpha}\right)
$$

Suppose now that

$$
h(z)=\left(z-e^{i \alpha_{1}}\right)^{\beta_{1}}\left(z-e^{i \alpha_{2}}\right)^{\beta_{2}} \cdots\left(z-e^{i \alpha_{k}}\right)^{\beta_{k}}
$$

where the $\alpha_{1}, \alpha_{2}, \cdots, \alpha_{k}$ are distinct real numbers, and where

$$
\sigma_{1}=\beta_{1}+\beta_{2}+\cdots+\beta_{k} \leqq 2 n \text {. }
$$

Write

$$
\begin{aligned}
\frac{z}{[h(z)]^{1 / n}}= & z^{1-\sigma / 2 n}\left[\frac{z}{\left(z-e^{i \alpha_{1}}\right)^{2}}\right]^{\beta_{1} / 2 n}\left[\frac{z}{\left(z-e^{i \alpha_{2}}\right)^{2}}\right]^{\beta_{2} / 2 n} \ldots \\
& \cdot\left[\frac{z}{\left(z-e^{i \alpha k}\right)^{2}}\right]^{\beta_{k} / 2 n} .
\end{aligned}
$$

Since the argument of the left side is the sum of the arguments of the factors on the right side, we see that the argument of the left side is nondecreasing (strictly increasing if $\sigma<2 n$ ) and that the total variation over the unit circle indented at the points $z=e^{i \alpha_{1}}, \cdots, e^{i \alpha_{k}}$ is equal to

$$
\left(1-\frac{\sigma}{2 n}\right) 2 \pi+\frac{1}{2 n}\left(\beta_{1}+\cdots+\beta_{k}\right) 2 \pi=2 \pi ;
$$

consequently, if $\sigma<2 n$, the function is schlicht.

If $\sigma=2 n$, then the unit circle is mapped on the whole plane except 
for the straight-line slits going from the points

$$
\frac{e^{-i \alpha_{j}}}{\left[h\left(e^{-i \alpha_{j}}\right)\right]^{1 / n}}, \quad j=1,2, \cdots, k \text { to } \infty,
$$

and therefore it is also schlicht. This proves the theorem.

\section{Additional results.}

COROLlaRY 1. If $h(z)$ is a polynomial with complex coefficients, satisfying the restrictions of Lemmas 2 and 3 , the function $f(z)$ $=z /[h(z)]^{1 / n}$ is schlicht for $z$ within the circle $|z|=1$.

This is true since the proof that $f(z)$ is schlicht, given in $\$ 3$, does not depend on the coefficients of $h(z)$ being integers.

Corollary 2. For $h(z)$, the polynomial of Corollary 1, and a any complex number except zero, the equation $h(z)=z^{n} / a^{n}$ has no more than $n$ distinct roots whose absolute values are less than 1 .

Let $\omega_{i}, i=1,2, \cdots, n$, be the $n$th roots of unity, and $z_{1}, z_{2}, \cdots, z_{m}$ the roots of the equation $h(z)=z^{n} / a^{n}$. For $z=z_{i}, i=1,2, \cdots, m$, the corresponding value of $f(z)$ on one of the sheets of its Riemann surface will be one of the $\omega_{j} a, j=1,2, \cdots, n$. Were there more than $n$ of the $z_{i}$ less than 1 in absolute value, then at least one of the $\omega_{j} a$ would correspond to two distinct values of $z$ within the unit circle and on the same sheet, contrary to the fact that $f$ is schlicht there.

\section{REFERENCES}

1. S. D. Bernardi, The theory of schlicht functions, Duke Math. J. vol. 19 (1952) pp. 263-287.

2. Bernard Friedman, Two theorems on schlicht functions, Duke Math. J. vol. 13 (1946) pp. 171-177.

3. H. Prawitz, Über Mittelwerte analytischer Funktionen, Arkiv för Matematik, Astronomi och Fysik vol. 20 (1927-1928) no. 6, pp. 1-12.

UNIVERSITY OF HAWAII 